\title{
Medical Image of the Week: Tracheal Perforation
}

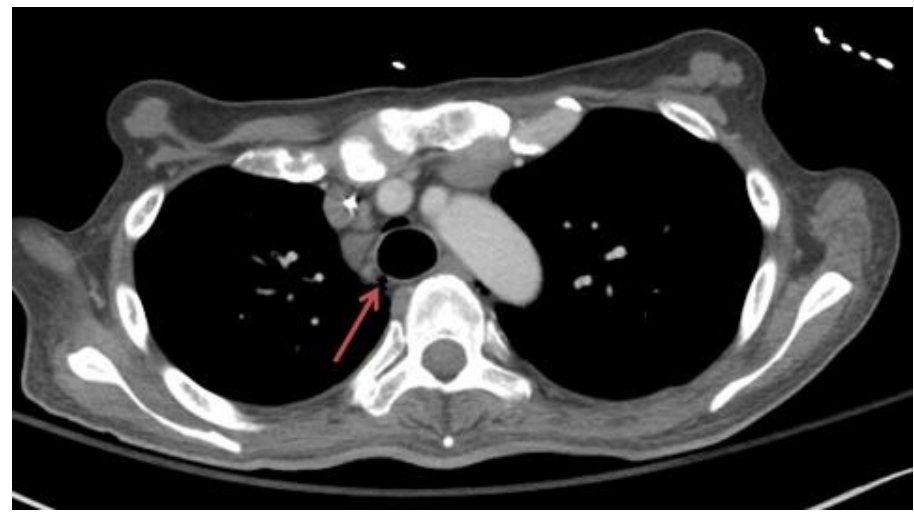

Figure 1. Axial thoracic CT scan showing air in the mediastinum (red arrow).

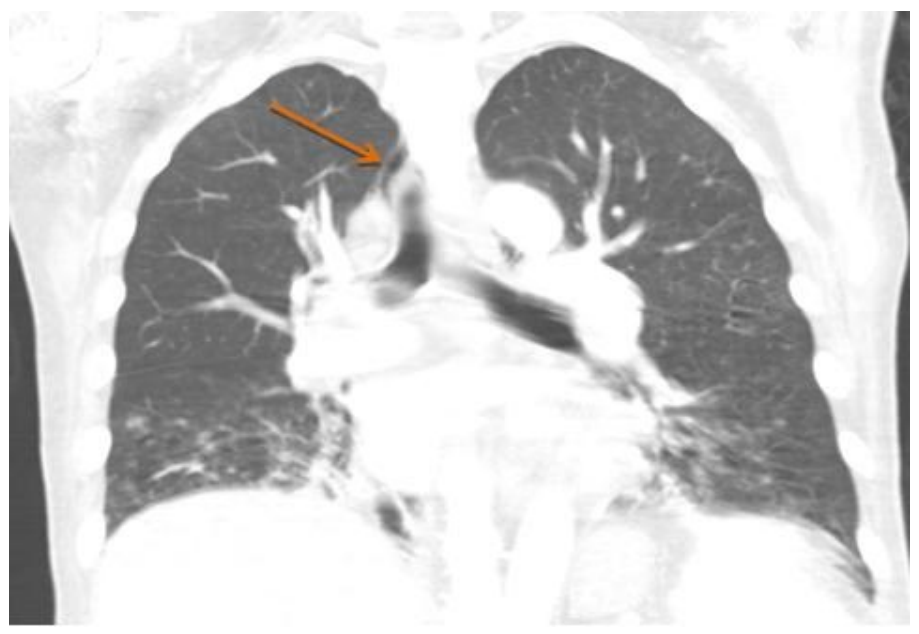

Figure 2. Coronal thoracic CT scan showing air in the mediastinum (orange arrow).

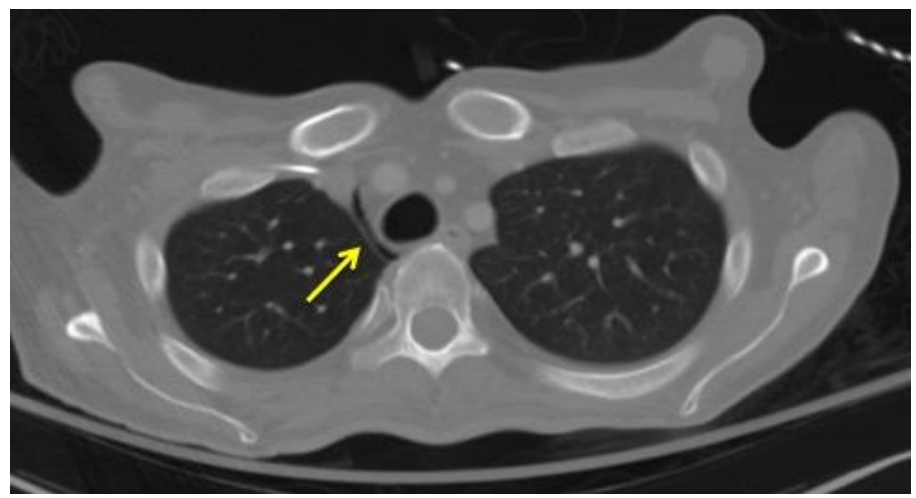

Figure 3. Axial thoracic CT scan showing air in the mediastinum (yellow arrow). 


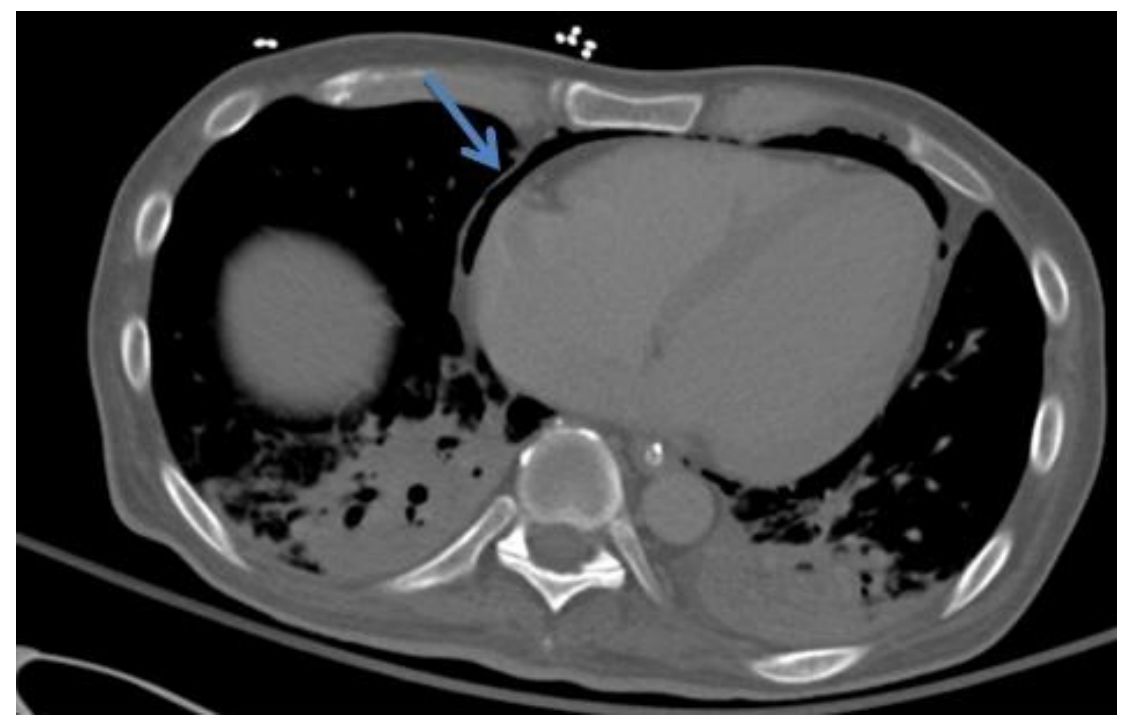

Figure 4. Axial thoracic CT scan showing pneumopericardium (blue arrow).

A 45 year old Caucasian man with a history of HIVIAIDS was admitted for septic shock secondary to right lower lobe community acquired pneumonia. The patient's respiratory status continued to decline requiring emergency intubation in a non-ICU setting. Four laryngoscope intubation attempts were made including an inadvertent esophageal intubation. Subsequent CT imaging revealed a tracheal defect (Figure 1, red arrow) with communication to the mediastinum and air around the trachea consistent with pneumomediastinum (Figure 2, orange arrow and figure 3, yellow arrow).

Pneumopericardium (figure 4, blue arrow) was also evident post-intubation. The patient's hemodynamic status remained stable. Two days following respiratory intubation subsequent chest imaging revealed resolution of the pneumomediastinum and pneumopericardium and patient continued to do well without hemodynamic compromise or presence of subcutaneous emphysema. Post-intubation tracheal perforation is a rare complication of traumatic intubation and may be managed with surgical intervention or conservative treatment (1).

Nour Parsa MD, Konstantin Mazursky DO, Sepehr Daheshpour MD, Naser Mahmoud MD

Department of Medicine

University of Arizona

Tucson, AZ

\section{Reference}

Fan CM, Ko PC, Tsai KC, Chiang WC, Chang YC, Chen WJ, Yuan A. Tracheal rupture complicating emergent endotracheal intubation. Am J Emerg Med. 2004;22(4):289-93. [CrossRef] [PubMed] 